\title{
Efeito do isolamento de árvores de Sterculia apetala sobre a emergência de plântulas no Pantanal
}

\author{
Antonio dos Santos Júnior ${ }^{1 *}$ \\ Walfrido Moraes Tomas ${ }^{2}$ \\ Marçal Henrique Amici Jorge ${ }^{3}$ \\ John Du Vall Hay ${ }^{4}$ \\ ${ }^{1}$ Instituto Federal de Educação, Ciência e Tecnologia de Rondônia \\ Avenida Jorge Teixeira, 3146, Setor Industrial, CEP 76821-002, Porto Velho - RO, Brasil \\ ${ }^{2}$ Laboratório de Vida Selvagem, Empresa Brasileira de Pesquisa Agropecuária \\ Embrapa Pantanal, Corumbá - MS, Brasil \\ ${ }^{3}$ Laboratório de Propagação de Plantas, Empresa Brasileira de Pesquisa Agropecuária \\ Embrapa Pantanal, Corumbá - MS, Brasil \\ ${ }^{4}$ Departamento de Ecologia, Instituto de Ciências Biológicas, Universidade de Brasília \\ Campus Universitário Darcy Ribeiro, Brasília - DF, Brasil \\ * Autor para correspondência \\ atonio.junior@ifro.edu.br
}

Submetido em 06/11/2012

Aceito para publicação em 09/07/2013

\section{Resumo}

Árvores de Sterculia apetala não são derrubadas durante a conversão de florestas em pastagens devido à consciência dos proprietários rurais de que essas árvores abrigam 95\% dos ninhos de arara-azul no Pantanal. Nosso objetivo foi comparar a porcentagem de emergência de plântulas em casa de vegetação de sementes de árvores em floresta conservada daquelas mantidas isoladas em pastagens cultivadas. As sementes foram coletadas diretamente sobre o solo, sob 10 árvores de $S$. apetala em cada área, no Pantanal, em Corumbá, Mato Grosso do Sul, Brasil. A emergência de plântulas de árvores isoladas foi 14\% inferior ao obtido para árvores do interior da floresta. É necessário que estudos futuros sobre a ecologia dessa espécie no Pantanal enfoquem o sistema reprodutivo, a identificação do agente polinizador e a avaliação do efeito do isolamento sobre a viabilidade populacional da espécie, a qual pode impactar o sucesso reprodutivo da arara-azul.

Palavras-chave: Arara-azul; Árvore ninho; Desflorestamento no Pantanal; Pastagem cultivada

\section{Abstract}

Effect of the isolation of Sterculia apetala trees on the emergence of seedlings in Pantanal. Sterculia apetala trees aren't removed during the conversion of forests into pastures due to rural landowners' awareness that these trees are home to $95 \%$ of the nests of Hyacinth Macaw in Pantanal. Our aim was to compare the greenhouse emergence rate of seedlings from seeds of trees at conserved forest and those left isolated in a cultivated pastures. The seeds were collected directly from the ground, under $10 \mathrm{~S}$. apetala trees in each area, at the Pantanal Wetland, Corumba, Mato Grosso do Sul, Brazil. The emergence of seedlings from isolated trees was $14 \%$ lower than that obtained from trees located in the inner part of the forest. There's a need for further studies 
on the ecology of this species in Pantanal focused on the mating system, the identification of the pollination agent and the evaluation of the effects of isolation on the viability of species population, which may impact the Hyacinth Macaw reproduction success.

Key words: Cultivated pasture; Deforestation in Pantanal; Hyacinth macaw; Nest tree

\section{Introdução}

Aárvore Sterculia apetala (Jacq.) Karst (Malvaceae) apresenta ampla distribuição geográfica na região Neotropical e por isso não está listada como ameaçada de extinção. Porém, pequenas populações em sua área de ocorrência têm sido destruídas pela substituição de florestas nativas por culturas comerciais, como pastagens para bovinos (JANZEN, 1972; DVORAK et al., 1998; SANTOS JR et al., 2007). No Pantanal Matogrossense as florestas nativas representavam $26 \%$ da cobertura do terreno (SILVA et al., 2000) e têm sido cumulativamente convertidas em pastagens de Brachiaria sp. (HARRIS et al., 2006).

A atividade pecuária bovina vem sendo desenvolvida no Pantanal há mais de 200 anos (MAZZA et al., 1994). Inicialmente era realizada de modo extensivo nos campos nativos, com os animais em baixa densidade, obedecendo ao ritmo do pulso anual de inundação. Nas últimas décadas, a partir de 1970, a atividade pecuária iniciou um novo modelo de produção, no qual os habitats florestais no Pantanal têm sido preferencialmente substituídos por campos de pastagens cultivadas, visando aumentar a densidade e a produtividade de gado bovino (COMASTRI-FILHO; POTT, 1995; JOHNSON et al., 1997; JUNK; SILVA, 1999).

Neste processo de conversão de florestas em pastagens no Pantanal, árvores de $S$. apetala não são derrubadas, preferencialmente as adultas e de grande porte, devido ao conhecimento dos proprietários de terras de que estas abrigam 95\% dos ninhos de arara-azul, Anodorhynchus hiacinthynus Latham 1720 (Psittacidae) (PINHO; NOGUEIRA, 2003; SANTOS JR, 2008). Esta ave está listada como ameaçada de extinção devido à captura de indivíduos para o comércio ilegal de animais silvestres e à destruição do seu habitat natural por queimadas ou desflorestamentos (NUNES et al., 2006). Esta prática tem dois efeitos sobre a estrutura populacional de $S$. apetala no Pantanal (dados não publicados). Primeiro, é alterada a distribuição etária pela remoção das plântulas e das árvores jovens onde há a introdução de pastagem. Em segundo, é reduzida a densidade populacional da espécie localmente, pois resta na população um número menor de indivíduos de S. apetala por hectares em relação à condição original de floresta.

As árvores poupadas permanecem isoladas do remanescente florestal envoltas por uma matriz artificial composta por pastagem (SANTOS JR, 2008) e sob tal circunstância é esperado que, como descrito para outras árvores de grande porte, sofram uma série de alterações nos processos que envolvem sua biologia reprodutiva (KAGEYAMA; PIÑA-RODRIGUES, 1993; MAUÉS; OLIVEIRA, 2010).

Agentes polinizadores como aves, mamíferos e insetos atuam para garantir a polinização biótica cruzada ao visitar flores de diferentes árvores de uma mesma espécie no intervalo de uma estação reprodutiva (PIÑA-RODRIGUES; PIRATELLI, 1993). Partindo do pressuposto de que o polinizador ocorre na floresta em que determinada espécies arbórea mantém sua população, a movimentação deste animal dependerá de estímulos visuais ou químicos por parte das plantas floridas que só podem ser percebidos até certa distância e, também, da inexistência de barreiras que dificultem ou impeçam a movimentação do polinizador (KAGEYAMA; PIÑA-RODRIGUES, 1993; PIÑA-RODRIGUES; PIRATELLI, 1993; MAUÉS; OLIVEIRA, 2010).

A matriz artificialmente gerada por atividades humanas, como a introdução de pastagens cultivadas em substituição às florestas nativas no Pantanal, apresenta-se como uma barreira à movimentação de alguns pássaros como Synallaxis albilora, Poecilotriccus latirostrae e Basileuterus flaveolus (NUNES, 2009). É razoável presumir que espécies que realizam os serviços de 
polinização também interpretem esta nova matriz no ecossistema como uma barreira comportamental. Neste sentido, algumas árvores isoladas em pastagens poderão ter menor probabilidade de visitação pelos polinizadores ou poderão estar mais sujeitas à autopolinização que suas coespecíficas que vivem no interior das florestas (LACERDA; KAGEYAMA, 2003; PETIT; HAMPE, 2006). Alguns autores argumentam que, em algumas circunstâncias, populações de árvores sofrem os efeitos da depressão endogâmica em caso de autopolinização, situação que pode ser constatada através da porcentagem de emergência e na formação de plântulas anormais (SEBBEN et al., 2000; ANDRADE et al., 2006), provocando uma redução na taxa de recrutamento de novas plantas na população.

Estudos que investiguem o efeito do isolamento de árvores sobre todos estes processos exigem um extenso trabalho de campo. Contudo, a investigação das etapas envolvidas na formação de sementes, bem como da dinâmica de como tais etapas estão interligadas, permitem a compreensão dos efeitos do isolamento de árvores com base na porcentagem de germinação, consumindo menos tempo e recursos de pesquisa e possibilitando a sugestão de diretrizes de conservação de uma dada população in situ.

O objetivo deste estudo foi investigar a diferença na porcentagem de emergência de plântulas geradas por sementes de $S$. apetala coletadas de árvores em florestas preservadas em comparação com aquelas isoladas em matriz de pastagens cultivadas no Pantanal.

\section{Material e Métodos}

Sterculia apetala é uma árvore neotropical de distribuição ampla, encontrada tanto em sítios bem drenados, com pronunciada estação seca de 4-6 meses (JANZEN, 1972; SAUTU et al., 2006), quanto em áreas de precipitação em torno de $3.000 \mathrm{~mm}$ por ano (FERNÁNDEZ et al., 2007).

Em diferentes sítios no Brasil a densidade estimada de árvores adultas de $S$. apetala é de menos de uma árvore com diâmetro na altura do peito $\geq 10$ cm por hectare (DUBS, 1992; PINTO; HAY, 2005; SALIS et al., 2006). Os indivíduos reprodutivos de
S. apetala são monoicos com as flores unissexuais na mesma inflorescência. As flores desta árvore são apétalas e monoclamídeas, exibindo exclusivamente um perianto carnoso, de cor branco-amarelada com detalhes em roxo e odor desagradável (POTT; POTT, 1994). Relatos sobre a biologia reprodutiva desta espécie não foram encontrados. Assim, os seus polinizadores são desconhecidos. Porém, observando a síndrome apresentada pelas flores (PIÑA-RODRIGUES; PIRATELLI, 1993), pode-se sugerir que os polinizadores sejam insetos, como besouros ou moscas, geralmente atraídos por flores com estas características.

Tomando por base os resultados discutidos por Sautu et al. (2006), relato no qual $S$. apetala foi uma das espécies que teve a longevidade das sementes investigadas, pode-se adotar a classificação proposta e assumir que esta espécie possui sementes longevas (ou não-recalcitrantes) por apresentar viabilidade de germinação por 15 meses quando estocadas em sacos de papel a $20{ }^{\circ} \mathrm{C}$ e $60 \%$ de umidade relativa do ar.

O clima da região de coleta das sementes é do tipo Aw de köppen, com uma estação seca, de Abril a Outubro e outra chuvosa, de Novembro a Março, apresentando uma média anual de pluviosidade de 1.200 mm e de temperatura $26^{\circ} \mathrm{C}$ (SORIANO, 1999). O relevo é plano com altitude aproximada de $90 \mathrm{~m}$ e os solos apresentam restrições de fertilidade para manejo agropastoril intensivo (SILVA; ABDON, 1998). A paisagem é predominantemente aberta, com campos inundáveis ou não e lagoas de água doce ou salobra intercaladas por manchas naturais de ambientes florestais (RATTER et al., 1988).

As sementes foram coletadas diretamente sobre o solo no período natural de dispersão (ver POTT; POTT, 1994), entre agosto e outubro de 2009, no Campo Experimental da Embrapa Pantanal (Fazenda Nhumirim, 1900'52"S; 56 $\left.38^{\circ} 38^{\prime \prime} \mathrm{W}\right)$ e na Fazenda Ipanema (1902'41'S - 56 $36^{\circ}$ '07'W), ambas localizadas na sub-região da Nhecolândia, Município de CorumbáMS (SILVA; ABDON, 1998). A primeira área de coleta é coberta por manchas de floresta estacional semidecidual, localmente chamadas de "cordilheiras" (RATTER et al., 1988; SILVA et al., 2000). A segunda área é composta por pastagens de Brachiaria decumbens e B. humidicola 
introduzidas em substituição à floresta original, com algumas árvores de $S$. apetala e de outras espécies mantidas isoladas na matriz de pastagens cultivadas, como Astronium fraxinifolium e Hymenaea courbaril.

A população de $S$. apetala em ambiente preservado estava contida no interior de uma unidade de conservação criada há mais de 20 anos, denominada RPPN Fazenda Nhumirim, condição extremamente incomum no Pantanal, onde as terras são predominantemente de propriedade privada e manejadas extensivamente para a produção pecuária, isto é, as florestas estão sujeitas aos eventos de incêndio florestal e pastejo por bovinos (JOHNSON et al., 1997; SEIDL et al., 2001). Devido à Fazenda Nhumirim não apresentar árvores de S. apetala isoladas em matriz de pastagem, optou-se por estudar uma população sob tais condições na Fazenda Ipanema. Nesta propriedade foi adotada a prática de conversão do sub-bosque e do dossel em pastagem cultivada, poupando algumas árvores emergentes.

$\mathrm{Na}$ Fazenda Nhumirim, as sementes foram coletadas sob a copa de 10 árvores de $S$. apetala que cresciam no interior de floresta estacional semidecidual nativa e sem evidências recentes de incêndio florestal ou remoção seletiva de madeira. Na Fazenda Ipanema, as sementes foram coletadas sob a copa de 10 árvores de $S$. apetala, todas isoladas na matriz de pastagem distantes pelo menos $200 \mathrm{~m}$ da floresta mais próxima. Foram coletadas no decorrer de dois meses 50 sementes de cada árvore, totalizando 500 sementes provenientes do ambiente florestal preservado e 500 da matriz de pastagem.

As sementes foram armazenadas por 80 dias em sacos de papel e em temperatura ambiente. Posteriormente, no Laboratório de Propagação de Plantas da Embrapa Pantanal, foram separadas 250 sementes perfeitamente sadias para cada tratamento. $\mathrm{O}$ ensaio foi realizado em casa de vegetação durante
30 dias, com a contagem de plântulas a cada três dias e irrigação distribuída quatro vezes ao dia para garantir umidade constante e uniforme. As sementes de cada tratamento foram distribuídas em dois blocos (tratamentos), com cinco repetições (réplicas) de 50 sementes cada. Todas as sementes foram depositadas deitadas e levemente comprimidas na superfície sem camada de cobertura em tubetes, contendo substrato para horticultura (Plantmax HT $\left.{ }^{\circledR}\right)$, dispostos em bandejas suspensas de arame galvanizado. Os dados foram transformados para $\log _{10}(\mathrm{x}+1)$ e submetidos ao teste ANOVA de um fator e dois níveis. No final do estudo, foram sorteadas e retiradas 20 plântulas de cada repetição, totalizando 100 plântulas de cada tratamento, das quais foi mensurada a altura, medindo-se a porção do colo até a gema apical, e o peso fresco da parte aérea. Os dados foram submetidos ao teste ANOVA de um fator e dois níveis. Todas as análises estatísticas foram realizadas com o pacote estatístico SYSTAT 11.

\section{Resultados}

No tratamento de floresta conservada, a primeira plântula emergiu aos 10 dias após a semeadura (DAS) e no de árvores isoladas aos 11 DAS. Até 20 DAS, a maioria das plântulas dos dois tratamentos avaliados havia emergido. $\mathrm{O}$ ensaio foi encerrado aos $28 \mathrm{DAS}$, quando a emergência das plântulas cessou por três observações consecutivas. Emergiram $82 \pm 5,3 \%$ das sementes das árvores na floresta e apenas $68 \pm 5,8 \%$ das sementes das árvores isoladas, valores que diferem significativamente $(F=14,062 ; p=0,006 ; \alpha=0,05)$

As plântulas do tratamento de floresta preservada apresentaram a maior altura $(\mathrm{F}=3,905 ; \mathrm{P}=0,048$; $\alpha=0,05)$ e maior peso fresco da parte aérea $(F=7,323$; $\mathrm{P}=0,007 ; \alpha=0,05$ ) ao final do experimento (Tabela 1 ).

TABELA 1: Altura e peso fresco da parte aérea de plântulas de Sterculia apetala após teste de germinação em casa de vegetação ( $N=100 /$ tratamento). Sementes coletadas em árvores crescendo em floresta ou isoladas em pastagens no Pantanal, Corumbá, MS.

\begin{tabular}{lccc|cccc}
\hline & \multicolumn{3}{c|}{ Altura $(\mathbf{c m})$} & \multicolumn{3}{c}{ Peso fresco da parte aérea $(\mathbf{g})$} \\
\cline { 2 - 7 } & Min & Med $\pm \mathbf{D P}$ & Max & Min & Med \pm DP & Max \\
\hline Floresta & 1,50 & $19 \pm 3,42$ & 28,00 & 3,69 & $6 \pm 0,88$ & 8,47 \\
Pastagem & 8,60 & $18 \pm 3,20$ & 25,60 & 1,38 & $5 \pm 1,55$ & 11,10 \\
\hline
\end{tabular}




\section{Discussão}

A emergência de plântulas de $S$. apetala estudada por Sautu et al. (2006) no Panamá ocorreu entre 12 e 27 DAS, padrão similar ao do presente estudo, a despeito das condições em que as árvores matrizes se encontravam. Para os dois tratamentos aqui avaliados a porcentagem de emergência foi aproximadamente o dobro da observada para esta espécie no Panamá ( $\sim 37 \%)$. Esta diferença pode ser explicada por diferenças ecológicas dos ambientes e também metodológicas, uma vez que Sautu et al. (2006) colheram as sementes de frutos diretamente da árvore ou sobre o solo, enquanto que, no presente estudo, as sementes foram colhidas na sombra da árvore após a dispersão primária, quando uma quantidade maior de sementes pode estar fisiologicamente madura (PIÑARODRIGUES; AGUIAR, 1993).

A emergência do tratamento de pastagem foi aproximadamente $14 \%$ inferior ao tratamento da floresta. Como as condições de germinação foram iguais para os dois tratamentos, uma hipótese para explicar esta diferença é a menor frequência de visitação de um dos agentes polinizadores às árvores isoladas (RANKINDE-MERONA; ACKERLY, 1987), dado o maior risco de predação quando comparado ao ambiente florestal (MAUÉS; OLIVEIRA, 2010). Nunes (2009) demonstra que a remodelação da paisagem pantaneira pelo desmatamento e introdução de pastagens cultivadas altera a mobilidade de algumas aves. Aplicando este raciocínio ao deslocamento na paisagem pelos agentes polinizadores de $S$. apetala, ainda desconhecidos, espera-se certa redução na produção e na qualidade de sementes em ambientes alterados, pois a ausência de um dos polinizadores ou a redução na sua frequência resultaria em uma grande quantidade de flores não polinizadas, impactando a fertilização dos óvulos e minorando a formação de sementes (KAGEYAMA; PIÑA-RODRIGUES, 1993). Portanto, sugerimos o desenvolvimento de estudos de biologia floral e de polinização para identificar os agentes polinizadores $S$. apetala no Pantanal e suas interações ecológicas.

Alguns dos polinizadores são capazes de visitar as flores de árvores de $S$. apetala isoladas na pastagem cultivada e esta ação pode promover a autofecundação, mais do que no interior da floresta, na qual a quantidade disponível de outras espécies com flores é maior. A população de árvores isoladas na pastagem pode sofrer depressão endogâmica, uma alteração no processo de fertilização do óvulo, resultando no aumento da mortalidade de sementes e desenvolvimento anormal de plântulas (SEBBEN et al., 2000). Porém, a deformação de plântulas não foi avaliada neste estudo.

Em recente revisão bibliográfica de Maués e Oliveira (2010), sobre as consequências da fragmentação do habitat na ecologia reprodutiva de espécies arbóreas em florestas tropicais, foram listados estudos demonstrando que em áreas desmatadas ocorre uma redução na quantidade de pólen depositado no estigma das flores, bem como uma redução da frutificação e da produção de sementes. Segundo os autores, é esperada a ocorrência de um aumento na autofecundação e diminuição no número de visitantes florais e polinizadores, especialmente em árvores isoladas em sistema agropastoris. Este padrão foi descrito para ecossistemas florestais que diferem marcadamente do Pantanal, que é um mosaico natural de campos, savanas e florestas (RATTER et al., 1988). Mesmo assim, aplicando as deduções deste modelo para o Pantanal, os resultados aqui apresentados são coerentes com a teoria ecológica vigente e permitem sugerir que a porcentagem de emergência de plântulas é menor para árvores de $S$. apetala isoladas em pastagens devido às condições restritivas aos processos de polinização (KAGEYAMA; PIÑA-RODRIGUES, 1993).

A compreensão sobre a dinâmica de florestas no Pantanal é incipiente. A ausência de estudos no âmbito deste ecossistema impossibilita a comparação dos resultados aqui relatados com outros na região. Os estudos realizados neste ecossistema enfocam especialmente a estrutura e composição de comunidades florestais. As respostas de populações aos diferentes fatores biológicos ou antrópicos precisam ser elucidadas. É necessário, por exemplo, que estudos futuros sobre S. apetala no Pantanal enfoquem o sistema reprodutivo e o fluxo gênico, identificando o agente polinizador e avaliando se o isolamento destas árvores em pastagens pode comprometer a manutenção de uma população viável da espécie e secundariamente ameaçar a reprodução da arara azul. 


\section{Agradecimentos}

Os autores agradecem ao Sr. João Monteiro por permitir a coleta das sementes na Fazenda Ipanema e aos revisores que contribuíram para incrementar a qualidade do relato apresentado. Os autores são gratos às contribuições dos revisores anônimos.

\section{Referências}

ANDRADE, A. C. S.; PEREIRA, T. S.; FERNANDES, M. J.; CRUZ, A. P. M.; CARVALHO, A. S. R. Substrato, temperatura de germinação e desenvolvimento pós-seminal de sementes de Dalbergia nigra. Pesquisa Agropecuária Brasileira, Brasília, v. 41, n. 3, p. 517-523, 2006.

COMASTRI-FILHO, J. A.; POTT, A. Introdução e avaliação de forragens em "cordilheiras" e "campo-cerrado" na parte leste da sub-região dos Paiaguás, Pantanal Mato-grossense. Pesquisa Agropecuária Brasileira, Brasília, v. 30, n. 8, p. 1117-1127, 1995. DUBS, B. Observation on the differentiation of woodland and wet savanna habitats in the Pantanal of Mato Grosso, Brazil. In FURLEY, P. A.; PROCTOR, J.; RATTER, J. A. (Org.). Nature and dynamics of forest-savanna boundaries. London: Chapman \& Hall, 1992. p. 417-429.

DVORAK, W. S.; UREÑA, H.; MORENO, L. A.; GOFORT, J. Provenance and family variation in Sterculia apetala in Colombia Forest Ecology and Management, Amsterdam, v. 111 p. 127-135, 1998.

FERNÁNDEZ, A.; COLONNELLO, G.; GUZMÁN, A. Inventario de la diversidad floristica de um sector Del curso médio Del rio Palmar. Revista de La Faculdad de Agronomia, Montevideo, v. 24, n. 1, p. 415-421, 2007.

HARRIS, M.; ARCANGELO, C.; PINTO, E. C.; CAMARGO, G.; RAMOS-NETO, M. B.; SILVA, S. M. Estimativa de perda de cobertura vegetal original na Bacia do Alto Paraguai e Pantanal Brasileiro: ameaças e perspectivas. Natureza \& Conservação, Curitiba, v. 4, n. 2, p. 50-66, 2006.

JANZEN, D. Escape in space by Sterculia apetala seeds from de bug Dysdercus fasciatus in a Costa Rican Deciduous Forest. Ecology, New York, v. 53, n. 2, p. 350-361, 1972.

JOHNSON, M. A.; TOMAS, W. M.; GUEDES, N. M. R. On the hyacinth macaw's nesting tree: density of young manduvis around adult trees under three different management conditions in the Pantanal wetland, Brazil. Ararajuba, São Paulo, v. 5, n. 2, p. $185-$ 188, 1997.

JUNK, W. J.; SILVA, C. J. O conceito do pulso de inundação e suas implicações para o Pantanal de Mato Grosso. In: SIMPÓSIO SOBRE RECURSOS NATURAIS E SÓCIO-ECONÔMICOS DO PANTANAL: MANEJO E CONSERVAÇÃO, II, 1999, Corumbá. Anais... Corumbá: Embrapa Pantanal, 1999. p. 17-28.

KAGEYAMA, P. Y.; PIÑA-RODRIGUES, F. C. M. Fatores que afetam a produção de sementes. In: AGUIAR, I. B.; PIÑARODRIGUES, F. C. M.; FIGLIOLIA, M. B. (Coord.). Sementes florestais tropicais. Brasília: ABRATES, 1993. p. 19-46.
LACERDA, C. M. B.; KAGEYAMA, P. Y. Estrutura genética espacial de duas populações naturais de Myracrodruon urundeuva M. Allemao na região semi-árida, Brasil. Revista Árvore, Viçosa, v. 27, n. 2, p. 145-150, 2003.

MAUÉS, M. M.; OLIVEIRA, P. E. A. M. Consequências da fragmentação do habitat na ecologia reprodutiva de espécies arbóreas em florestas tropicais, com ênfase na Amazônia. Oecologia Australis, Rio de Janeiro, v. 14, n. 1, p. 238-250, 2010.

MAZZA, M. C. M.; MAZZA, C. A. S.; SERENO, J. R. B.; SANTOS, S. A.; PELLEGRIN, A. O. Etnobiologia e conservação do bovino pantaneiro. Brasília: EMBRAPA-SPI, 1994. 320 p.

NUNES, A. P. Ocupação de manchas florestais por três espécies de aves insetívoras do sub-bosque no Pantanal da Nhecolândia, Corumbá, Mato Grosso do Sul. 2009. 71 f. Dissertação (Mestrado em Ecologia) - Universidade Federal de Mato Grosso do Sul, Campo Grande. 2009.

NUNES, A. P.; TIZIANEL, F. A. T.; TOMAS, W. M. Aves ameaçadas ocorrentes no Pantanal. Corumbá: EMBRAPA Pantanal, 2006. 47 p. (Série Documentos no 83)

PETIT, R. J.; HAMPE, A. Some evolutionary consequences of being a tree. Annual Review Ecology, Evolution and Systematics, Palo Alto, v. 37, p. 187-214, 2006.

PIÑA-RODRIGUES, F. C. M.; AGUIAR, I. B. Maturação e dispersão de sementes. In: AGUIAR, I. B.; PIÑA-RODRIGUES, F. C. M.; FIGLIOLIA, M. B. (Coord.). Sementes florestais tropicais. Brasília: ABRATES, 1993. p. 83-135.

PIÑA-RODRIGUES, F. C. M.; PIRATELLI, A. J. Aspectos ecológicos da produção de sementes. In: AGUIAR, I. B.; PIÑARODRIGUES, F. C. M.; FIGLIOLIA, M. B. (Coord.). Sementes florestais tropicais. Brasília: ABRATES, 1993. p. 47-81.

PINHO, J. B.; NOGUEIRA, F. M. B. Hyacinth macaw (Anodorhynchus hyacinthinus) reproduction in the Northern Pantanal, Mato Grosso, Brazil. Ornitologia Neotropical, Bonn, v 14, n. 2, p. 29-38, 2003.

PINTO, J. R. R.; HAY, J. D. Mudanças florísticas e estruturais na comunidade arbórea de uma floresta de vale no Parque Nacional da Chapada dos Guimarães, Mato Grosso, Brasil. Revista Brasileira de Botânica, São Paulo, v. 28, n. 3, p. 523-539, 2005.

POTT, A.; POTT, V. J. Plantas do Pantanal. Brasília: EMBRAPA. 1994. 320 p.

RANKIN-DE-MERONA，J. M.; ACKERLY，D. D. Estudos populacionais de árvores em florestas fragmentadas e as implicações para a conservação "in situ" das mesmas na floresta tropical da Amazônia Central. Instituto de Pesquisas e Estudos Florestais, Piracicaba, v. 35, p. 47-59, 1987.

RATTER, J. A.; POTT, A.; POTT, V. J.; NUNES DA CUNHA, C.; HARIDASAN, M. Observation on Woody vegetation types in the Pantanal and at Corumbá, Brazil. Notes of Royal Botanic Garden Edinburg, Edinburg, v. 45, n. 3, p. 503-525, 1988.

SALIS, S. M.; ASSIS, M. A.; CRISPIM, S. M. A.; CASAGRANDE, J. C. Distribuição e abundância de espécies arbóreas em cerradões no Pantanal, Estado de Mato Grosso do Sul, Brasil. Revista Brasileira de Botânica, São Paulo, v. 29, n. 3, p. 339-352, 2006.

SANTOS JR, A. Do the results of ecological research contribute for biodiversity conservation of the Pantanal? The Hyacinth Macaw case. Natureza \& Conservação, Curitiba, v. 6, n. 2, p. 133-138, 2008. 
SANTOS JR, A.; TOMAS, W. M.; ISHII, I. H.; GUEDES, N. M. R.; HAY, J. D. Occurrence of Hyacinth Macaw nesting sites in Sterculia apetala in the Pantanal Wetland, Brazil. Gaia Scientia, João Pessoa, v. 1, n. 2, p. 127-130, 2007.

SAUTU, A.; BASKIN, J. M.; BASKIN, C. C.; CONDIT, R. Studies on the seed biology of 100 native species of trees in a seasonal moist tropical forest, Panamá, Central America. Forest Ecology and Management, Amsterdam, v. 234, p. 245-263, 2006.

SEBBEN, A. M.; KAGEYAMA, P. Y.; SIQUEIRA, A. C. M. F.; ZANATTO, A. C. S. Sistema de cruzamento em populações de Cariniana legalis Mart. O. Ktze: implicações para a conservação e o melhoramento genético. Scientia Forestalis, Piracicaba, v. 58, p. $25-40,2000$.
SEIDL, A. F.; SILVA, J. S. V.; MORAES, A. S. Cattle ranching and deforestation in the Brazilian Pantanal. Ecological Economics, Amsterdam, v. 36, p. 413-426. 2001.

SILVA, J. S. V.; ABDON, M. M. Delimitação do Pantanal brasileiro e suas sub-regiões. Pesquisa Agropecuária Brasileira, Brasília, v. 33, n. 1, p. 1703-1711, 1998.

SILVA, M. P.; MAURO, R.; MOURÃO, G.; COUTINHO, M. Distribuição e quantificação de classes de vegetação do Pantanal através de levantamento aéreo. Revista Brasileira de Botânica, São Paulo, v. 23, n. 1, p. 143-152, 2000.

SORIANO, B. M. A. Caracterização climática da sub-região da Nhecolândia, Pantanal, MS. In: SIMPÓSIO SOBRE RECURSOS NATURAIS E SÓCIO-ECONÔMICOS DO PANTANAL: MANEJO E CONSERVAÇÃO, II, 1999, Corumbá. Anais... Corumbá: Embrapa Pantanal, 1999. p. 151-158. 